\title{
CHIRAL-ODD GENERALIZED PARTON DISTRIBUTIONS, TRANSVERSITY AND DOUBLE TRANSVERSE-SPIN ASYMMETRY IN DRELL-YAN DILEPTON PRODUCTION*
}

\author{
M. Pincetti ${ }^{\ddagger}$, B. Pasquini, S. Boffi \\ Dip. di Fisica Nucleare e Teorica, Università degli Studi di Pavia \\ and INFN, Sezione di Pavia, \\ Pavia, 27100, Italy \\ ${ }^{\ddagger}$ E-mail: manuel.pincetti@pv.infn.it
}

\begin{abstract}
Within the framework of light-cone quantization we derive the overlap representation of generalized parton distributions for transversely polarized quarks using the Fock-state decomposition in the transverse-spin basis. We apply this formalism to the case of light-cone wave functions in a constituent quark model giving numerical results for the four chiral-odd generalized parton distributions in a region where they describe the emission and reabsorption of a quark by the nucleon. With the transversity distribution obtained in the forward limit of the generalized distribution, we provide some predictions for the double transverse-spin asymmetry in Drell-Yan dilepton production in the kinematics of the $\mathcal{P} \mathcal{A X}$ experiment.

Keywords: Generalized Parton Distributions; Transversity; Drell-Yan; Double Transverse-Spin Asymmetry
\end{abstract}

\section{Introduction}

Quarks and gluons are the fundamental degrees of freedom in the study of strong interactions by means of QCD. Nevertheless, the objects experimentally observable are hadrons and, at present, we have not yet a complete description of how hadrons are built up in terms of quark and gluon fields. In hard scattering processes we parameterize this non perturbative information through a set of parton distribution (PDs), which are hadronic matrix elements of bilocal products of the light-front quark and gluon field operators. At leading twist a quark-parton model of the nucleon requires three PDs: the unpolarized distribution, $f_{1}(x)$, the helicity distribution, $g_{1}(x)$,

*This research is part of the EU Integrated Infrastructure Initiative Hadronphysics Project under contract number RII3-CT-2004-506078. 
and the transversity distribution, $h_{1}(x)$. Whereas the first two distributions are well studied physical quantities, both from the experimental and theoretical point of view, so far, the last one is totally unknown. As a matter of fact, $h_{1}(x)$ does not contribute to inclusive deep-inelastic scattering because of its chiral-odd nature and is only experimentally accessible when coupled to another chiral-odd partner in the cross section. Notwithstanding, some theoretical activity has been developed to calculate it and to suggest some new experimental ways to extract $h_{1}(x)$ from data ${ }^{1}$. Among all the proposed experimental investigations, the favorite one is represented by the double transversely polarized Drell-Yan dilepton production ${ }^{2}$. In fact, this allows the study of the double transverse-spin asymmetry of lepton-pair production, giving direct access to the transversity distribution.

In recent years a novel class of parton distributions has been introduced, the generalized parton distributions (GPDs) ${ }^{3}$. The GPDs have attracted a considerable amount of interest, since it has been pointed out that they contain a wealth of information about the internal structure of hadrons, interpolating between the inclusive physics of parton distributions and the exclusive limit of form factors ${ }^{4}$. These new parton distributions functions are defined as non-diagonal hadronic matrix elements of bilocal products of the light-front quark and gluon field operators. A complete set of quark GPDs at leading twist include four helicity conserving, usually labeled $H$, $E, \tilde{H}, \tilde{E}$, and four helicity flipping chiral-odd GPDs, labeled $H_{T}, E_{T}, \tilde{H}_{T}$, $\tilde{E}_{T}{ }^{5,6}$. In the forward limit, $H, \tilde{H}$ and $H_{T}$ reduce to $f_{1}, g_{1}$ and $h_{1}$, respectively.

A variety of model calculations is available for helicity conserving GPDs. Less attention has been paid up to now to the chiral-odd sector. So far, there is only one proposal to access the chiral-odd GPDs in diffractive double meson production ${ }^{7,8}$. In this paper we study the chiral-odd GPDs by means of the overlap representation of light-cone wave functions (LCWFs) that was originally proposed in Refs. ${ }^{9}$. In a fully covariant approach the connection between the overlap representation of GPDs and the non-diagonal one-body density matrix in momentum space has further been explored in Refs. ${ }^{10}$ for the helicity conserving sector, making use of the correct transformation of the wave functions from the (canonical) instant-form to the (light-cone) front-form description. In this way the lowest-order Fock-space components of LCWFs with three valence quarks are directly linked to wave functions derived in constituent quark models (CQMs). Recently this approach has been extended to include the next-order Fock-state component, by developing a convolution formalism for the unpolarized GPDs which 
incorporate the sea quark distribution ${ }^{11}$.

In this paper, following the approach discussed in Refs. ${ }^{12,13}$, the four chiral-odd GPDs are derived in Sec. 2 by means of a Fock-state decomposition in the transverse spin basis and with LCWFs for three valence quarks. In Sec. 3 we present the numerical results obtained for the GPDs, while we show our estimations for the transversity distributions in Sec. 4 and some predictions for the double transverse-spin asymmetry in Drell-Yan dilepton production in Sec. 5 .

\section{The Overlap Representation}

The chiral-odd GPDs are off-diagonal in the parton helicity basis. In the reference frame where the momenta $\vec{p}$ and $\vec{p}^{\prime}$ of the initial and final nucleon lie in the $x-z$ plane, they become diagonal if one changes basis from eigenstates of helicity to eigenstates of transversity, i.e. states with spin projection $\uparrow(\downarrow)$ directed along (opposite to) the transverse direction $\hat{x}$. In such a basis they are obtained by means of the following relations

$$
\begin{aligned}
H_{T}^{q}= & \frac{1}{\sqrt{1-\xi^{2}}} T_{\uparrow \uparrow}^{q}-\frac{2 M \xi}{\epsilon \sqrt{t_{0}-t}\left(1-\xi^{2}\right)} T_{\uparrow \downarrow}^{q}, \\
E_{T}^{q}= & \frac{2 M \xi}{\epsilon \sqrt{t_{0}-t}} \frac{1}{1-\xi^{2}} T_{\uparrow \downarrow}^{q}+\frac{2 M}{\epsilon \sqrt{t_{0}-t}\left(1-\xi^{2}\right)} \tilde{T}_{\uparrow \uparrow}^{q} \\
& -\frac{4 M^{2}}{\left(t_{0}-t\right) \sqrt{1-\xi^{2}}\left(1-\xi^{2}\right)}\left(\tilde{T}_{\downarrow \uparrow}^{q}-T_{\uparrow \uparrow}^{q}\right), \\
\tilde{H}_{T}^{q}= & \frac{2 M^{2}}{\left(t_{0}-t\right) \sqrt{1-\xi^{2}}}\left(\tilde{T}_{\downarrow \uparrow}^{q}-T_{\uparrow \uparrow}^{q}\right), \\
\tilde{E}_{T}^{q}= & \frac{2 M}{\epsilon \sqrt{t_{0}-t}\left(1-\xi^{2}\right)}\left(T_{\uparrow \downarrow}^{q}+\xi \tilde{T}_{\uparrow \uparrow}^{q}\right) \\
& -\frac{4 M^{2} \xi}{\left(t_{0}-t\right) \sqrt{1-\xi^{2}}\left(1-\xi^{2}\right)}\left(\tilde{T}_{\downarrow \uparrow}^{q}-T_{\uparrow \uparrow}^{q}\right),
\end{aligned}
$$

where $t=\Delta^{2}$ is the transferred momentum square, $-t_{0}=4 m^{2} \xi^{2} /\left(1-\xi^{2}\right)$ is its minimum value for a given value of the skewness parameter $\xi=$ $-\Delta^{+} / 2 P^{+}$defined in terms of the momentum transfer $\Delta^{\mu}=p^{\prime \mu}-p^{\mu}$ and the average nucleon momentum $P^{\mu}=\frac{1}{2}\left(p+p^{\prime}\right)^{\mu}$, and $\epsilon=\operatorname{sign}\left(D^{1}\right)$, where $D^{1}$ is the $x$-component of $D^{\alpha}=P^{+} \Delta^{\alpha}-\Delta^{+} P^{\alpha}$. 
The transverse matrix elements are defined as follows

$$
\begin{aligned}
& T_{\lambda_{t}^{\prime} \lambda_{t}}^{q}=\left\langle p^{\prime}, \lambda_{t}^{\prime}\left|\int \frac{d z^{-}}{2 \pi} e^{i \bar{x} P^{+} z^{-}} \bar{\psi}(-z / 2) \gamma^{+} \gamma^{1} \gamma_{5} \psi(z / 2)\right| p, \lambda_{t}\right\rangle, \\
& \tilde{T}_{\lambda_{t}^{\prime} \lambda_{t}}^{q}=\left\langle p^{\prime}, \lambda_{t}^{\prime}\left|\int \frac{d z^{-}}{2 \pi} e^{i \bar{x} P^{+} z^{-}} \frac{i}{2} \bar{\psi}(-z / 2) \sigma^{+1} \psi(z / 2)\right| p, \lambda_{t}\right\rangle .
\end{aligned}
$$

In the region $\xi \leq \bar{x} \leq 1$ of plus-momentum fractions, where the generalized quark distributions describe the emission of a quark with plus-momentum $(\bar{x}+\xi) P^{+}$and its reabsorption with plus-momentum $(\bar{x}-\xi) P^{+}$, they can be written in the overlap representation of LCWFs as

$$
\begin{aligned}
T_{\lambda_{t}^{\prime} \lambda_{t}}^{q}= & \sum_{N, \beta=\beta^{\prime}}(\sqrt{1-\xi})^{2-N}(\sqrt{1+\xi})^{2-N} \sum_{j=1}^{N} \operatorname{sign}\left(\mu_{j}^{t}\right) \delta_{s_{j} q} \\
& \times \int[d \bar{x}]_{N}\left[d^{2} \vec{k}_{\perp}\right]_{N} \delta\left(\bar{x}-\bar{x}_{j}\right) \Psi_{\lambda_{t}^{\prime}, N, \beta^{\prime}}^{*}\left(\hat{r}^{\prime}\right) \Psi_{\lambda_{t}, N, \beta}(\tilde{r}), \\
\tilde{T}_{\lambda_{t}^{\prime} \lambda_{t}}^{q}= & \sum_{\beta, \beta^{\prime}, N}(\sqrt{1-\xi})^{2-N}(\sqrt{1+\xi})^{2-N} \sum_{j=1}^{N} \delta_{\mu_{j}^{t^{\prime} \prime}-\mu_{j}^{t}} \delta_{\mu_{i}^{t^{\prime}} \mu_{i}^{t}} \operatorname{sign}\left(\mu_{j}^{t}\right) \delta_{s_{j} q} \\
& \times \int[d \bar{x}]_{N}\left[d^{2} \vec{k}_{\perp}\right]_{N} \delta\left(\bar{x}-\bar{x}_{j}\right) \Psi_{\lambda_{t}^{\prime}, N, \beta^{\prime}}^{*}\left(\hat{r}^{\prime}\right) \Psi_{\lambda_{t}, N, \beta}(\tilde{r}),
\end{aligned}
$$

where $\Psi_{\lambda_{t}, N, \beta}$ is the momentum LCWF of the $N$-parton Fock state, $s_{j}$ labels the quantum numbers of the $j$-th active parton, with transverse initial (final) spin polarization $\mu_{j}^{t}\left(\mu_{j}^{t^{\prime}}\right)$, and $\mu_{i}^{t}\left(\mu_{i}^{t^{\prime}}\right)$ are the transverse spin of the spectator initial (final) quarks. The set of kinematical variables $\tilde{r}, \hat{r}^{\prime}$ are defined according to Refs. ${ }^{9,12}$.

\section{Results}

As an application of the general formalism we consider the valence-quark contribution $(N=3)$ to the chiral-odd GPDs with the LCWF derived starting from an instant-form of the proton obtained in the relativistic quark model of Ref. ${ }^{14}$. The structure of the nucleon wave function in this model is $\mathrm{SU}(6)$ symmetric for the spin-isospin components. As an example we show in Fig. 1 the four calculated chiral-odd GPDs, $H_{T}^{u}, E_{T}^{u}, \tilde{H}_{T}^{u}, \tilde{E}_{T}^{u}$ at $t=-0.2(\mathrm{GeV})^{2}$ and for different values of $\xi$. In all cases the GPDs vanish at $\bar{x}=\xi$ since in our approach they include the contribution of valence quarks only and we cannot populate the so-called ERBL region with $|\bar{x}| \leq \xi$ where quark-antiquark pairs and gluons are important. Therefore, at low $\bar{x}$ this gives a strong $\xi$ dependence of the peak position of the distribution, but for large $\bar{x}$ the $\xi$ dependence turns out to be rather weak. 

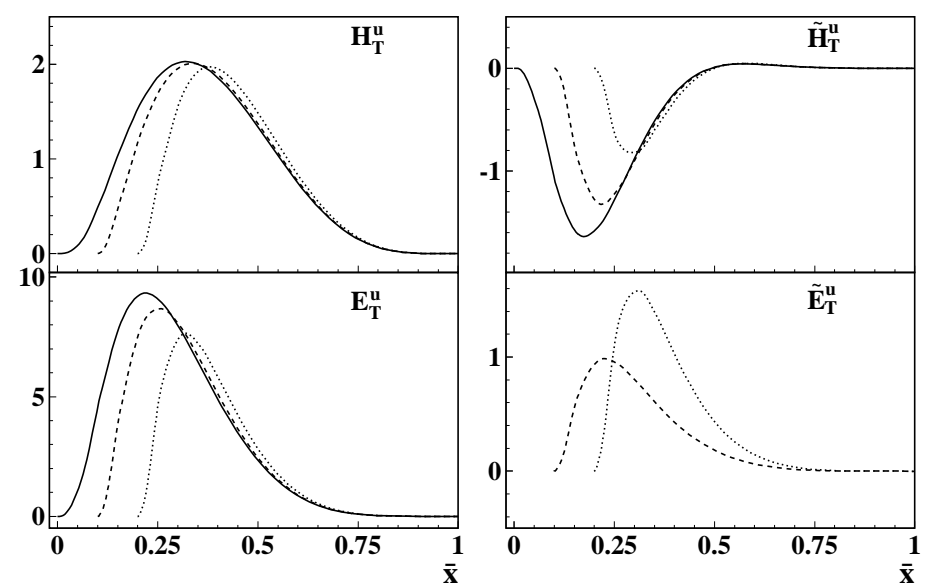

Figure 1. The chiral odd generalized parton distributions calculated in the CQM of Ref. ${ }^{14}$ for the flavour $u$ at $t=-0.2(\mathrm{GeV})^{2}$ and for different values of $\xi: \xi=0$ (solid curves), $\xi=0.1$ (dashed curves), $\xi=0.2$ (dotted curves).

Concerning the $t$ dependence, it affects the low- $\bar{x}$ region and is more pronounced in the cases of $E_{T}^{q}$ and $\tilde{H}_{T}^{q}$. For large $\bar{x}$ values the decay of all the distributions towards zero at the boundary $\bar{x}=1$ is almost independent of $t$ (see Ref. ${ }^{12}$ ).

\section{The Forward Limit}

In the forward limit $\Delta^{\mu} \rightarrow 0(\bar{x} \rightarrow x$, with $x$ being the usual Bjorken variable), only the quark GPD $H_{T}^{q}$ can be measured and, in fact, becomes the quark transversity distribution $h_{1}^{q}(x)$. Although the quark GPDs $E_{T}^{q}$ and $\tilde{H}_{T}^{q}$ do not contribute to the scattering amplitude, they remain finite in the forward limit, whereas $\tilde{E}_{T}^{q}$ vanishes identically being an odd function of $\xi$ as already noticed in Ref. ${ }^{6}$.

The transversity distribution $h_{1}^{q}$ is the counterpart in the transversepolarization space of the helicity parton distribution $g_{1}^{q}$ which measures the helicity asymmetry. As it was stressed by $\mathrm{Jaffe}$ and $\mathrm{Ji}^{2}$, in nonrelativistic situations where rotational and boost operations commute, one has $g_{1}^{q}=h_{1}^{q}$. Therefore the difference between $h_{1}^{q}$ and $g_{1}^{q}$ is a measure of the relativistic nature of the quarks inside the nucleon. In light-cone CQMs these relativistic effects are encoded in the Melosh rotations.

In Fig. 2 the helicity and transversity distributions, $g_{1}$ and $h_{1}$, obtained as a forward limit of the corresponding GPDs calculated with the CQM of Ref. ${ }^{14}$, are compared and plotted together with the nonrelativistic result 

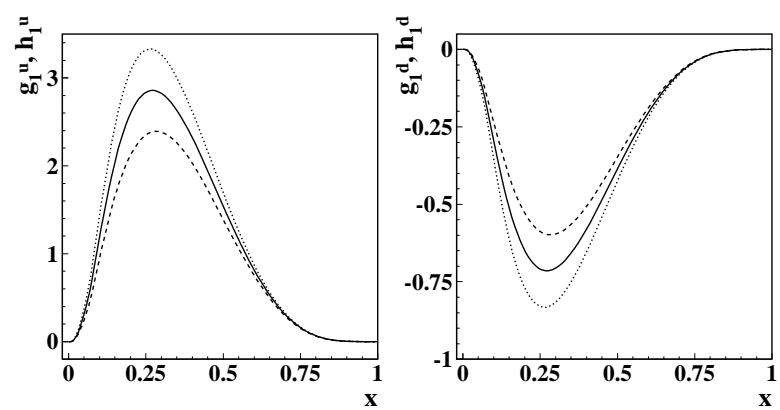

Figure 2. Helicity and transversity distributions for the $u$ (left panel) and $d$ (right panel) quark. The solid lines correspond to $h_{1}^{q}$, the dashed lines show $g_{1}^{q}$, and the dotted lines are the nonrelativistic results when Melosh rotations reduce to the identity $\left(h_{1}^{q}=g_{1}^{q}\right)$.

when Melosh rotations reduce to identity. The large difference between $g_{1}$ and $h_{1}$ shows how big is the effect of relativity.

Recalling the expression for the unpolarized parton distribution $f_{1}^{q}$ obtained in Ref. ${ }^{10}$, it is easy to see that the following relations hold

$$
2 h_{1}^{u}(x)=g_{1}^{u}(x)+\frac{2}{3} f_{1}^{u}(x), \quad 2 h_{1}^{d}(x)=g_{1}^{d}(x)-\frac{1}{3} f_{1}^{d}(x),
$$

which are compatible with the Soffer inequality ${ }^{15}$. In the nonrelativistic limit one obtains $h_{1}^{u}=g_{1}^{u}=2 / 3 f_{1}^{u}$ and $h_{1}^{d}=g_{1}^{d}=-1 / 3 f_{1}^{d}$.

\section{The Double Transverse-Spin Asymmetry}

In order to directly access transversity via Drell-Yan lepton pair production one has to measure the double transverse-spin asymmetry $A_{T T}$ in collisions between two transversely polarized hadrons:

$$
A_{T T}=\frac{\mathrm{d} \sigma(\uparrow \uparrow)-\mathrm{d} \sigma(\uparrow \downarrow)}{\mathrm{d} \sigma(\uparrow \uparrow)+\mathrm{d} \sigma(\uparrow \downarrow)}
$$

with the arrows denoting the transverse directions along which the two colliding hadrons are polarized. The most favorable situation for a sizeable effect is the process $p^{\uparrow} \bar{p}^{\uparrow} \rightarrow l^{+} l^{-} X$ mediated by a virtual photon ${ }^{16}$. At LO, i.e. considering only the quark-antiquark annihilation graph, the double transverse-spin asymmetry for this process is given by

$$
A_{T T}^{p \bar{p}}=a_{T T} \frac{\sum_{q} e_{q}^{2}\left[h_{1}^{q}\left(x_{1}, Q^{2}\right) h_{1}^{q}\left(x_{2}, Q^{2}\right)+h_{1}^{\bar{q}}\left(x_{1}, Q^{2}\right) h_{1}^{\bar{q}}\left(x_{2}, Q^{2}\right)\right]}{\sum_{q} e_{q}^{2}\left[f_{1}^{q}\left(x_{1}, Q^{2}\right) f_{1}^{q}\left(x_{2}, Q^{2}\right)+f_{1}^{\bar{q}}\left(x_{1}, Q^{2}\right) f_{1}^{\bar{q}}\left(x_{2}, Q^{2}\right)\right]},
$$


where $e_{q}$ is the quark charge, $Q^{2}$ the invariant mass square of the lepton pair (dimuon), and $a_{T T}$ the spin asymmetry of the QED elementary process $q \bar{q} \rightarrow l^{+} l^{-}$. Here, as in Ref. ${ }^{13}$, we provide quantitative estimates

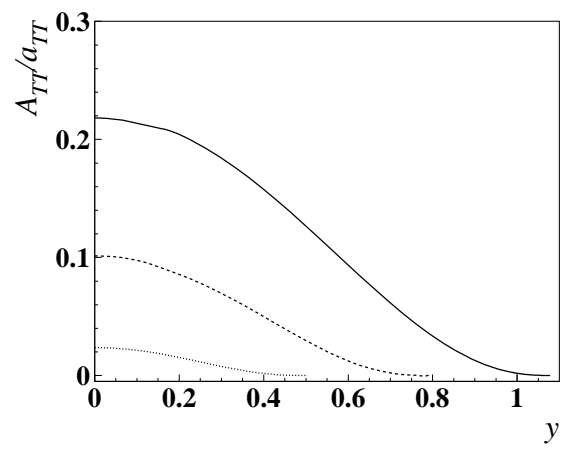

Figure 3. The $A_{T T}^{p \bar{p}} / a_{T T}$ at a center of mass energy square of $45 \mathrm{GeV}^{2}$ and different values of $Q^{2}: 5 \mathrm{GeV}^{2}$ (solid line), $9 \mathrm{GeV}^{2}$ (dashed line), $16 \mathrm{GeV}^{2}$ (dotted line).

of $A_{T T}^{p \bar{p}} / a_{T T}$ for the kinematics of the proposed $\mathcal{P} \mathcal{A X}$ experiment at GSI. In Fig. 3 our predictions for $A_{T T}^{p \bar{p}} / a_{T T}$ are plotted in terms of the rapidity $y=1 / 2 \ln \left(x_{1} / x_{2}\right)$, where the transversity distribution has been suitably evolved solving numerically the DGLAP equations ${ }^{17}$ (see Fig. 4 ) and where the unpolarized distribution functions $f_{1}\left(x, Q^{2}\right)$ are taken from the GRV parameterization $^{18}$.

One may notice that our predictions for $A_{T T}^{p \bar{p}} / a_{T T}$ are much lower than the results obtained in others phenomenological analysis ${ }^{16}$. However, our study suggests the possibility of a measurable asymmetry, about $20 \%$, at moderate $Q^{2}$ values, around $5 \div 10 \mathrm{GeV}^{2}$, giving direct access to the transversity distribution.

\section{Bibliography}

1. V. Barone, A. Drago, P.G. Ratcliffe, Phys. Rep. 359, 1 (2002); V. Barone, P.G. Ratcliffe, Transverse spin physics (World Scientific, Singapore, 2003).

2. J. Ralston, D. Soper, Nucl. Phys. B 152, 109 (1979); R.L. Jaffe, X. Ji, Phys. Rev. Lett. 67, 552 (1991); Nucl. Phys. B 375, 527 (1992); J.L. Cortes, B. Pire, and J.P. Ralston, Z. Phys. C 55, 409 (1992); X. Ji, Phys. Lett. B 284, 137 (1992); P. Lenisa et al. ( $\mathcal{P} \mathcal{A} \mathcal{X}$ Coll.), hep-ex/0505054; A. Bianconi, M. Radici, Phys. Rev. D 72, 074013 (2005).

3. D. Müller et al., Fortsch. Phys. 42, 101 (1994); A.V. Radyushkin, Phys. Lett. 

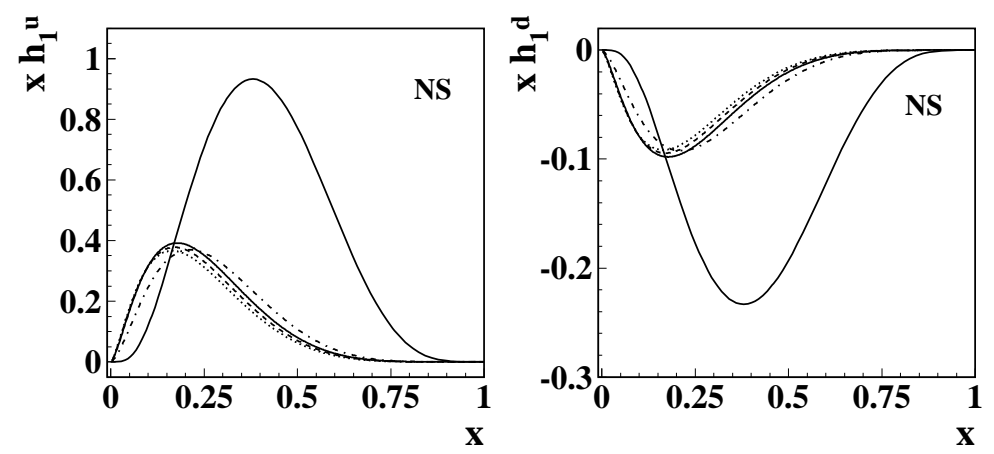

Figure 4. Evolution of the transversity distribution for the $u$ (left panel) and $d$ (right panel) quark. Starting from the hadronic scale of the model $Q_{0}^{2}=0.079 \mathrm{GeV}^{2}$ (upper curve), we plot the LO curves at different scales: $Q^{2}=5 \mathrm{GeV}^{2}$, solid curves; $Q^{2}=9$ $\mathrm{GeV}^{2}$, dashed curves; $Q^{2}=16 \mathrm{GeV}^{2}$, dotted curves, and a NLO curve at $Q^{2}=5 \mathrm{GeV}^{2}$ dashed-dotted curve.

B 380 (1996) 417; Phys. Lett. B 385, 333 (1996); X. Ji, Phys. Rev. Lett. 78, 610 (1997); J. Phys. G 24, 1181 (1998).

4. K. Goeke et al., Prog. Part. Nucl. Phys. 47, 401 (2001); M. Diehl, Phys. Rep. 388, 41 (2003); X. Ji, Ann. Rev. Nucl. Part. Sci. 54, 413 (2004); A.V. Belitsky, A.V. Radyushkin, Phys. Rep. 418, 1 (2005).

5. P. Hoodbhoy, X. Ji, Phys. Rev. D 58, 054006 (1998).

6. M. Diehl, Eur. Phys. J. C 19, 485 (2001).

7. B. Pire et al., Phys. Lett. B 550, 65 (2002); Phys. Part. Nucl. 35, 67 (2004).

8. R. Enberg, B. Pire, L. Szymanowski, Eur. Phys. J. C 47, 87 (2006).

9. M. Diehl et al., Eur. Phys. J. C 8, 409 (1999); Nucl. Phys. B 596, 33 (2001); S.J. Brodsky et al., Nucl. Phys. B 596, 99 (2001).

10. S. Boffi, B. Pasquini, M. Traini, Nucl. Phys. B 649, 243 (2003); Nucl. Phys. B 680, 147 (2004); Phys. Rev. D 71, 034022 (2005).

11. B. Pasquini, S. Boffi, Phys. Rev. D 73, 094001 (2006).

12. B. Pasquini, M. Pincetti, S. Boffi, Phys. Rev. D 72, 094029 (2006); M. Pincetti, B. Pasquini, S. Boffi, hep-ph/0610051.

13. B. Pasquini, M. Pincetti, S. Boffi, hep-ph/0612094.

14. F. Schlumpf, J. Phys. G 20 (1994) 237.

15. J. Soffer, Phys. Rev. Lett. 74, 1292 (1995).

16. A. Efremov et al., Eur. Phys. J. C 35, 207 (2004); M. Anselmino et al., Phys. Lett. B 594, 97 (2004); V. Barone et al., Phys. Lett. B 639, 483 (2006).

17. Yu.L. Dokshitzer, JETP (Sov. Phys.) 46, 641 (1977); V.N. Gribov, L.N. Lipatov, Sov. J. Nucl. Phys. 15, 438 (1972); G. Altarelli, G. Parisi, Nucl. Phys. $B$ 126, 298 (1977).

18. M. Glück, E. Reya, A. Vogt, Eur. Phys. J. C 5, 461 (1998). 